\title{
Understanding holographic photopolymer-dispersed liquid crystals for data storage
}

Inmaculada Pascual, Sergi Gallego, Andrés Márquez, Manuel Ortuño, Jorge Francés, and Augusto Beléndez

Interferometry at the zero spatial frequency limit and analysis of diffracted orders for very low spatial frequency gratings reveal the factors that control grating formation.

Holography is a technique to obtain optical elements (diffraction gratings, lenses, or mirrors), as well as data storage devices, by controlling both the phase and intensity of light. For these applications, the recording material must be optimized for efficient diffraction (i.e., with low scattering) and maximum information storage. Photopolymer materials are inexpensive, self-processing, have the ability to record low-loss, highdiffraction-efficiency optical elements, and also have high data storage capability, and thus are of interest for holographic recording media. In general, these materials absorb light of the appropriate wavelength, causing photopolymerization of the local monomer, inducing a change in the material refractive index and thickness.

Among photopolymeric materials, holographic polymer dispersed liquid crystals (H-PDLCs) are some of the most interesting. To obtain H-PDLCs, we mix liquid crystal molecules with photopolymer. ${ }^{1}$ The liquid crystals change their refractive index when an external electric field is applied. When this type of material is exposed to an interference pattern, local differences in photopolymerization rates induce periodic phase separation of discrete liquid crystal molecules, commensurate with the period of the interference pattern. When such a mixture is exposed to the optical field of two or more interfering laser beams, photopolymerization occurs more rapidly in the bright than in the dark regions of the interference pattern.

Two transformations-polymerization and diffusion-take place inside the H-PDLC during recording. We can characterize such a material by examining its behavior. Holographic recording materials are usually characterized by recording optical

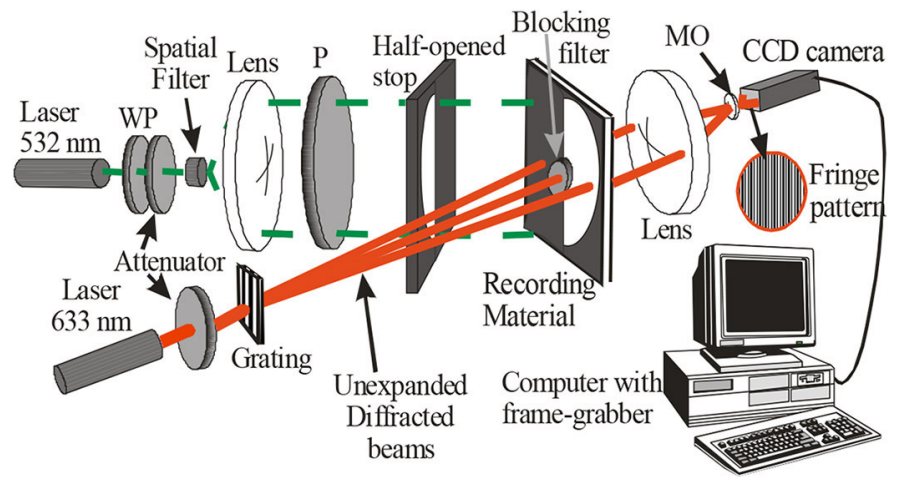

Figure 1. Experimental setup for zero spatial frequency analysis. P: Polarizer. WP: Wave plate. MO: Microscope objective.

elements as diffraction gratings ${ }^{2}$ (periodic structures that split the light beam into several beams, called orders, traveling in different directions). We have used two methods to characterize H-PDLC: an interferometric and a diffractive method. The main advantages of these methods are that characterization and optimization of the material, and recording holographic gratings, are performed simultaneously, and we obtain real-time information on polymerization and diffusion. The interferometric method enables us to analyze the influence of polymerization in the absence of diffusion, and the diffractive method provides information on the effect of diffusion in the absence of polymerization. By combining the two, we can understand the influence of different components, such as the liquid crystal molecules, on grating formation.

The first method is based on an interferometer that has been used to characterize liquid crystal displays (LCDs) by their phase shift versus applied voltage: see Figure $1 .^{3}$ We used a diffraction grating with a spatial frequency of four lines per millimeter to generate a series of diffracted orders and blocked all 
the orders except +1 and -1 . One of the two orders overlaps the exposed zone (illuminated by a 532nm laser), and the other overlaps the non-exposed zone. At the sample, the distance between the two orders is approximately $1 \mathrm{~cm}$, which eliminates the influence of monomer diffusion in the polymerization process. Afterwards, the orders are brought to interfere. From the interference pattern we can determine what happens inside the material due to polymerization. Diffusion does not occur.

For the photopolymer we used dipentaerythritol penta/hexaacrylate as monomer and binder, $\mathrm{N}$-vinyl pyrrolidone as crosslinker, ethyl eosin as dye, and $\mathrm{N}$-methyl diethanolamine as radical generator. ${ }^{4}$ To characterize the material, we used layers $42 \mu \mathrm{m}$ thick to increase the phase shift, which allows us to view more clearly the influence of the liquid crystal concentration.

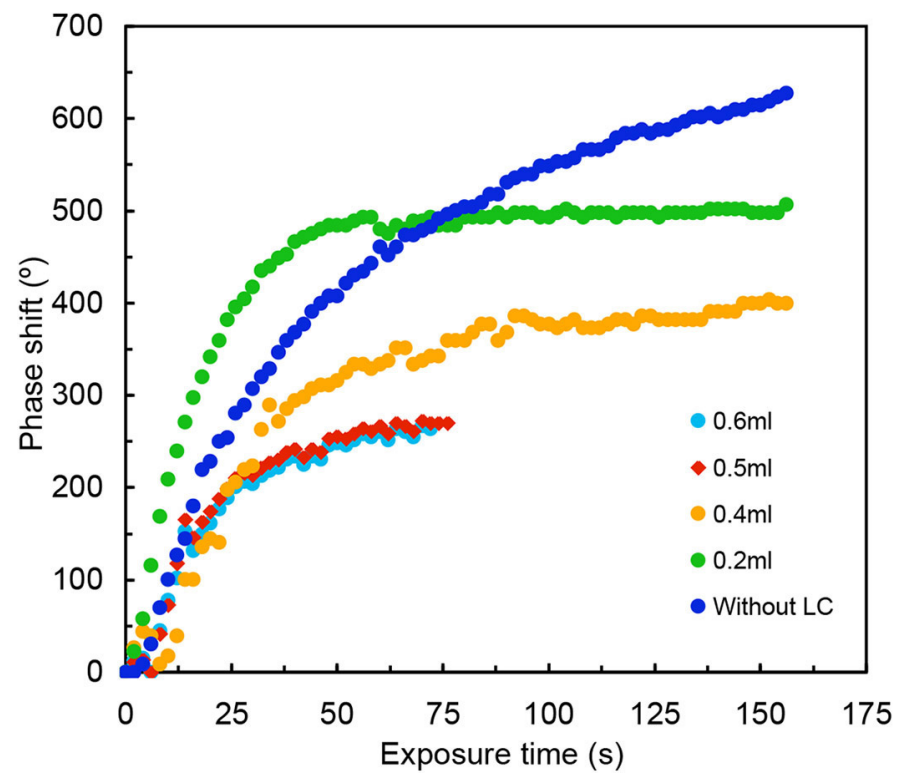

Figure 2. Behaviors of photopolymer at the zero spatial frequency limit in transmission for different quantities of liquid crystal (LC) molecules.

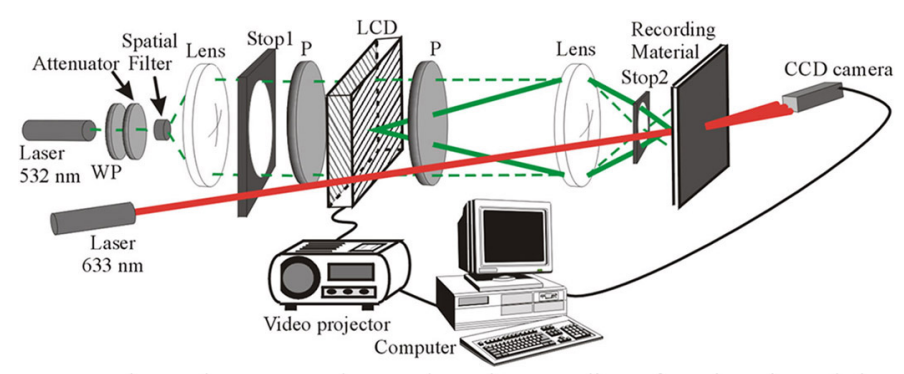

Figure 3. Experimental setup used to analyze the recording of gratings in real time. LCD: Liquid crystal display.

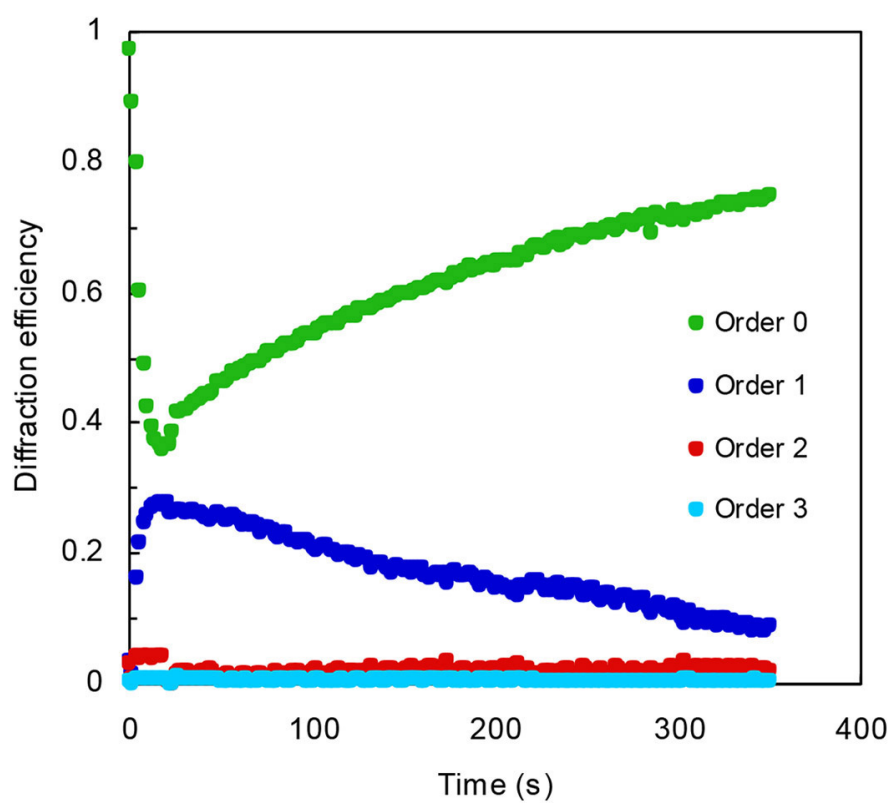

Figure 4. Diffraction intensities of the first four orders for a grating with spatial period of $0.168 \mathrm{~mm}$.

We used this interferometric method to analyze the influence of the dispersed liquid crystal concentrations on the polymerization process: see Figure 2. The phase shift (which is related to the polymer refractive index ${ }^{4}$ ) decreases from 600 to $250^{\circ}$ as the concentration of liquid crystal molecules increases.

In the second, 'diffractive,' method, for very low spatial frequency analysis, we use an LCD as the 'master' that is copied onto the photopolymer by laser illumination: see Figure 3. Polymerization takes place only where the light transmitted by the LCD meets the photpolymer. We used a solid-state laser (532nm), and the LCD was used in the 'amplitude-mostly' modulation regime. (Amplitude and phase are coupled. Thus, to obtain pure amplitude modulation requires a previous study of the LCD properties.) Using the LCD allowed us to change the period of the grating recorded in the photopolymer without moving any mechanical part of the setup. We used an unexpanded beam of a helium-neon laser $(633 \mathrm{~nm})$ incident at a small angle to the normal to the recording material to analyze in real time the variation in efficiency of the different diffraction orders.

We were able to measure more than eight diffracted orders and obtain reliable information about the phase profile recorded in the material and its temporal evolution after recording due to diffusion. Figure 4 shows the diffraction efficiency of the first four orders (a measure of the energy that can be obtained from 


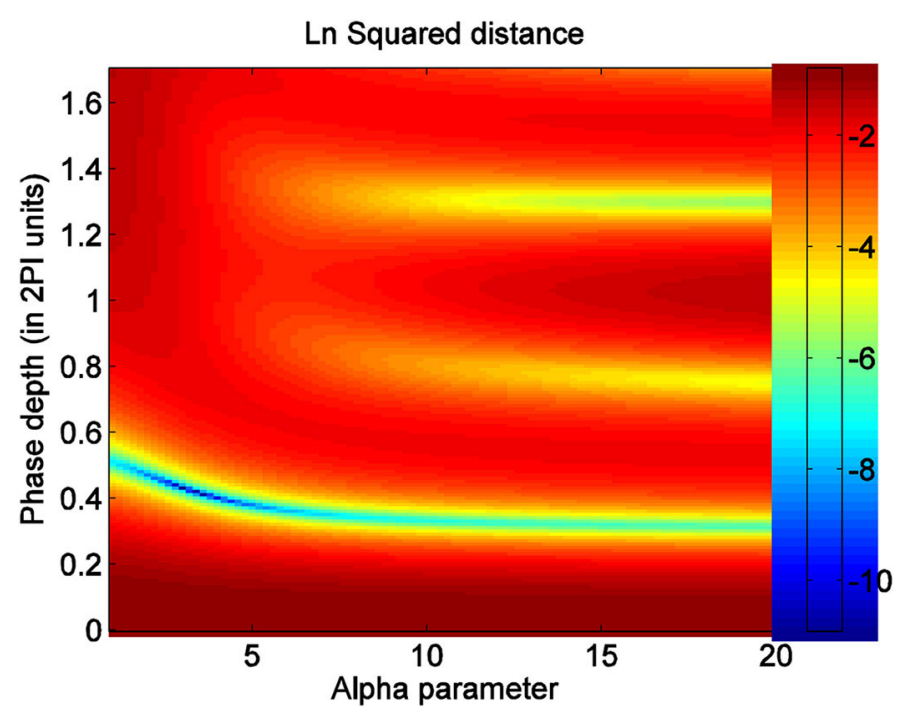

Figure 5. Search for the optimum phase profile after 16 s exposure. Alpha: A free parameter of the Fermi-Dirac distribution used to fit the numerical to the experimental results. The darker colors represent a smaller minimum square distance between the numerical and experimental results and consequently a better fit. Ln: Napierian logarithm.

each order, compared to the energy of the incident light). The 1st order achieves a diffraction efficiency of $28 \%$ just when the recording stops (i.e., at time $t=20 \mathrm{~s}$ ), and decreases after exposure. When the illuminating laser stops, diffusion returns all the energy to the 0th order. Consequently, the curve of the 0th order increases and the curve of the 1st order decreases after $t=20 \mathrm{~s}$. The contributions of the 2 nd and 3rd orders are much less than the contribution of the 1st order.

After measuring the diffracted efficiency of the main orders as a function of time, we used the Fermi-Dirac function to obtain the profile of the generated grating, and especially the sharpness of the edges and the phase-modulation depth or shape profile. ${ }^{5}$

The symmetrical Fermi-Dirac distribution has two free parameters, $\alpha$, and the phase depth: ${ }^{5}$ see Figure 5 for a representation of how the minimum square distance (the best fit between the theoretical and experimental values), shown by the darker (blue) zone, is found for a time exposure of 16s. The FermiDirac fittings provide important information that can be used to calculate the average species diffusion inside the material. ${ }^{4} \mathrm{By}$ fitting the numerical results (theoretical results) to the experimental ones, we can sketch the grating profile and thus also evaluate the importance of species diffusion for the shape of the edges.

In conclusion, using interferometric and diffractive analysis we characterized a photopolymer with and without liquid crystal molecules. By combining the two methods, we were able to analyze polymerization and diffusion separately and thus consider the influence of different components such as liquid crystal molecules on grating formation. Polymerization and diffusion are both fundamental parameters for understanding and simulating the generation of diffractive optical elements, holograms, or even optical memories when using H-PDLCs. Using some simple models, it is possible to fit the polymerization rate and the average species diffusion within the material. We are now studying the capability of the material as a holographic memory by storing several gratings in an H-PDLC and characterizing the holographic material.

This work was supported by the Spanish Ministry of Science and Innovation (FIS2011-29803-C02-01 and FIS2011-29803-C02-02) and by the Regional Government of Valencia, Spain, under projects PROMETEO/2011/021, ISIC/2012/013, and ACOMP/2013/075.

\section{Author Information}

\section{Inmaculada Pascual, Sergi Gallego, Andrés Márquez,} Manuel Ortuño, Jorge Francés, and Augusto Beléndez University of Alicante

Alicante, Spain

Inmaculada Pascual is a professor of optics. She received her MSc in physics at the University of Granada, Spain, and her $\mathrm{PhD}$ in physics at the University of Valencia, Spain. Her research interests include holographic materials, holographic optical elements, optical storage, and visual optics. In these fields she has published 120 journal papers, and 100 conference presentations. She is a senior member of SPIE and is coauthor of more than 50 SPIE proceedings papers. She has also participated in several SPIE conferences.

Sergi Gallego obtained his degree in physics from the University of Valencia in 2001 and his PhD in 2005 at the University of Alicante, where he is a lecturer. His research concerns holographic recording materials, LCDs applied to holography, diffractive elements, and photopolymers.

Andrés Márquez is an associate professor of applied physics. He received his MSc and $\mathrm{PhD}$ in physics from the Autonomous University of Barcelona, Spain. His research interests include holographic materials, liquid crystal spatial light modulators, and diffractive optics. He is a member of SPIE. 
Manuel Ortuño is a professor. He received an MS in organic chemistry and chemical engineering in 1999 and a PhD in physics in 2005. His areas of interest are photopolymers, holographic recording materials, liquid crystals, and green materials.

Jorge Francés was born in Alcoy, Spain, in 1982. He received his $\mathrm{PhD}$ in 2011 from the University of Alicante, where he has been working as an assistant lecturer since 2008. His main research interests include physical optics, sound and vibration, and numerical simulation.

Augusto Beléndez is a professor of applied physics. He received his MSc and PhD in physics from the University of Valencia. His research interests include holographic recording materials, holographic optical elements, and optical storage. He is a senior member of SPIE.
References

1. K. Curtis and D. Psaltis, Characterization of the DuPont photopolymer for 3dimensional holographic storage, Appl. Opt. 33 (23), pp. 5396-5399, 1994.

2. K. R. Sun, Y. S. Kang, and B. K. Kim, Transflective multiplexing of holographic polymer dispersed liquid crystal using Si additives, Exp. Polym. Lett. 5 (1), pp. 73-81, 2011. doi:10.3144/expresspolymlett.2011.8

3. A. J. Bergeron, F. Gauvin, D. Gagnon, H. Gingras, H. H. Arsenault, and M. Doucet, Phase calibration and applications of a liquid crystal spatial light modulator, Appl. Opt. 34, pp. 5133-5139, 1995. doi:10.1364/AO.34.005133

4. S. Gallego, A. Márquez, M. Ortuño, J. Francés, I. Pascual, and A. Beléndez, Diffractive and interferometric methods to characterize photopolymers with liquid crystal molecules as holographic recording material, J. Eur. Opt. Soc. 7, p. 12024, 2012 doi:10.2971/jeos.2012.12024

5. S. Gallego, A. Márquez, D. Méndez, S. Marini, A. Beléndez, and I. Pascual, Spatial-phase-modulation-based study of polyvinyl-alcohol/acrylamide photopolymers in the low spatial frequency range, Appl. Opt. 48, pp. 4403-4413, 2009. doi:10.1364/AO.48.004403 